\title{
Psychische Beschwerden - Neues aus der Rechtsprechung
}

\section{Marco Weiss}

Dr. iur., Teichmann International (Schweiz) AG, Anwaltskanzlei und Notariat, St. Gallen/Zürich/Frauenfeld

\author{
Das Bundesgericht hat in zwei aktuellen Leitentscheiden seine jahrelange und \\ höchst umstrittene Rechtsprechung zu den psychischen Beschwerdebildern geän- \\ dert. Nach BGE 143 V 418 und BGE 143 V 409 ist bei psychischen Leiden in Form einer \\ Depression nicht mehr allein die Diagnose entscheidend, sondern inwiefern sich \\ das diagnostizierte Leiden auf die Arbeitsfähigkeit der betroffenen Person tatsäch- \\ lich limitierend auswirkt.
}

\section{Einleitung}

In seiner aktuellen Rechtsprechung änderte das Bundesgericht seine jahrelange Praxis, nach welcher Depressionen leichter- bis mittelgradiger Natur keinen Anspruch auf eine Invalidenrente begründen konnten. Gemäss heutiger Rechtslage ist bei psychischen Leiden in Form einer Depression nicht mehr allein die Diagnose entscheidend, sondern inwiefern sich das diagnostizierte Leiden auf die Arbeitsfähigkeit der betroffenen Person tatsächlich leistungslimitierend auswirkt. Aus diesem Grund ist seit den Leitentscheiden BGE 143 V 418 und BGE 143 V 409 bei sämtlichen psychischen Gesundheitsleiden - wie bei den psychosomatischen Beschwerdebildern - ein strukturiertes Beweisverfahren anhand der vom Bundesgericht in BGE 141 V 281 genannten Indikatoren durchzuführen. Ausnahmen von diesem strukturierten Beweisverfahren sind nur in besonderen Ausnahmefällen möglich.

\section{Alte Rechtslage}

Gemäss der früheren Rechtsprechung des Bundesgerichts waren die Folgen einer depressiven Problema-

\section{Résumé}

Dans deux arrêts de principe rendus récemment, leTribunal fédéral a modifié sa jurisprudence de longue date très controversée en matière de troubles psychiques. Selon I'ATF 143 V 418 et l'ATF 143 V 409, l'élément déterminant en cas de troubles psychiques sous la forme d'une dépression n'est plus le diagnostic seul, mais la mesure dans laquelle l'affection diagnostiquée a effectivement un effet limitatif sur la capacité de travail de la personne concernée. tik nur bei überwiegend wahrscheinlicher Therapieresistenz von invalidenversicherungsrechtlicher Bedeutung. Nach der alten Rechtsprechung fielen depressive Störungen leicht- bis mittelgradiger Natur, unabhängig davon, ob sie rezidivierend oder episodisch waren, einzig dann als invalidisierende Krankheiten in Betracht, wenn sie erwiesenermassen als therapieresistent anzusehen waren [1]. Oder in anderen Worten ausgedrückt: Ein sozialversicherungsrechtlicher Leistungsanspruch aus der Invalidenversicherung war mit der Diagnose einer leicht- bis mittelgradigen Depression so gut wie ausgeschlossen, weil ein Anspruch nur bei ausgewiesener Therapieresistenz geltend gemacht werden konnte.

Die oben geschilderte Rechtslage wurde sowohl aus medizinischer als auch juristischer [2] Sicht kritisch betrachtet. Kritisiert wurde vor allem, dass aus der Behandelbarkeit, für sich allein betrachtet, nicht auf den invalidisierenden Charakter einer psychischen Störung geschlossen werden könne [3].

\section{Neue Rechtslage}

Das Bundesgericht unterzog seine alte Rechtsprechung in den Grundsatzentscheiden BGE 143 V 418 und BGE 143 V 409 einer genauen Prüfung und stellte sich die wesentliche Frage: Ist die Rechtsprechung, wonach depressive Störungen leicht- bis mittelgradiger Natur einzig dann als invalidisierende Krankheiten in Betracht fallen, wenn sie erwiesenermassen therapieresistent sind, aufzugeben? [4].

Das Bundesgericht begründete diese Rechtsprechungsänderung im Wesentlichen mit den folgenden Argumenten: Zuerst mit dem Aspekt, dass die bundes- 


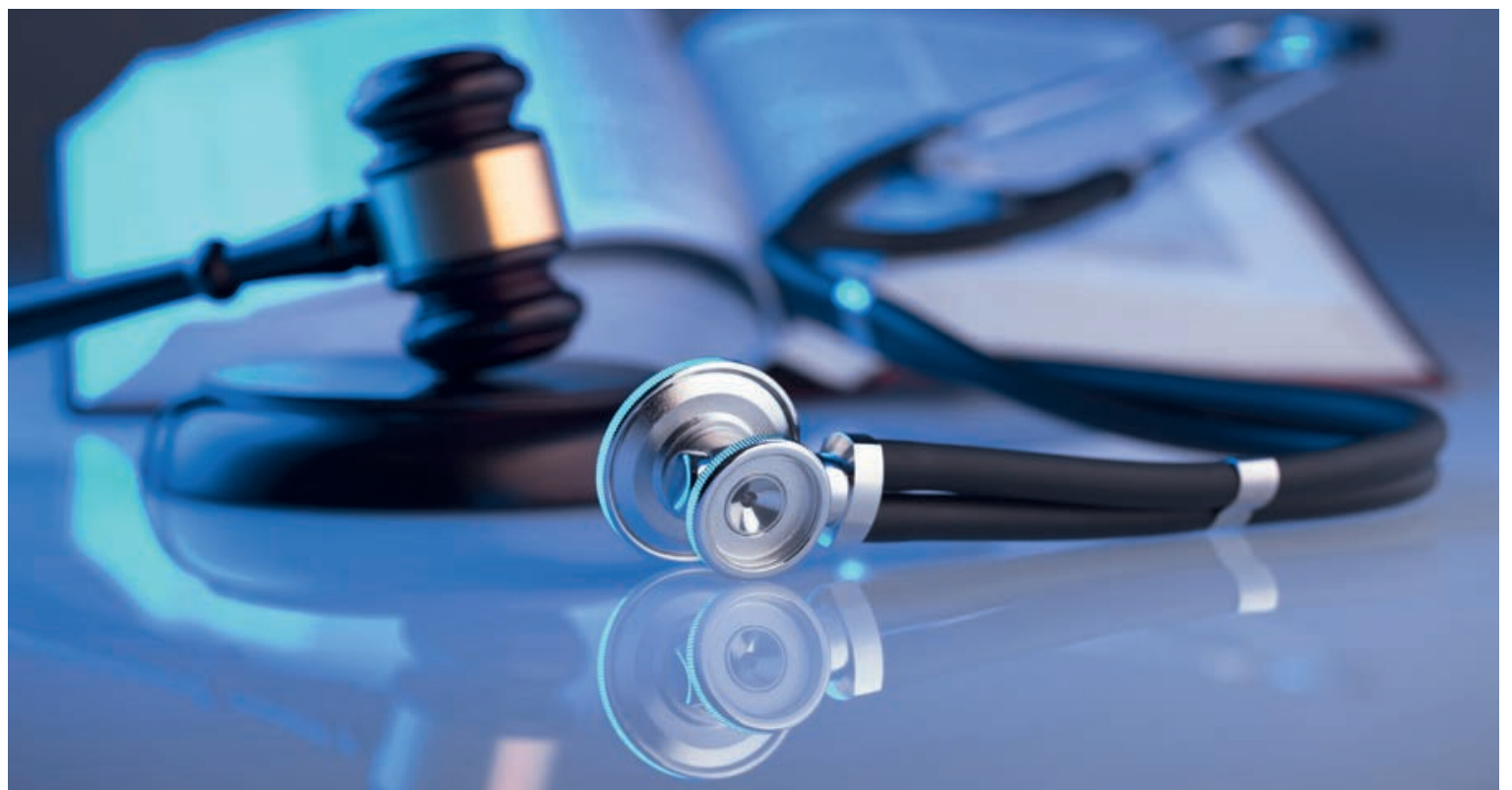

Bislang begründeten leichte- bis mittelgradige Depressionen keinen Anspruch auf eine Invalidenrente. Doch gemäss aktueller Rechtsprechung des Bundesgerichts ist bei psychischen Leiden in Form einer Depression nicht mehr allein die Diagnose entscheidend, sondern inwiefern sich das Leiden auf die Arbeitsfähigkeit der betroffenen Person tatsächlich leistungslimitierend auswirkt.

gerichtliche Rechtsprechung den Eintritt einer (rentenbegründenden) Invalidität nie gesamthaft von der Therapierbarkeit eines Leidens abhängig gemacht habe. Entscheidend sei die Frage, ob es der gesundheitlich beeinträchtigten Person zumutbar sei, eine Arbeitsleistung zu erbringen, was sich nur nach einem weitgehend objektivierten Massstab beurteilen lasse [5]. Diese dargelegten Grundsätze seien im Lichte des Grundsatzentscheides BGE 141 V 281 zu betrachten. Nach diesem Entscheid findet bei somatischen Schmerzstörungen eine Anspruchsprüfung anhand eines Indikatorenkatalogs statt, in welchem die Aspekte von Behandlungserfolg oder -resistenz (in der Kategorie «funktioneller Schweregrad») und ergänzend dazu, mit Blick auf den anamnestisch ausgewiesenen Leidensdruck, die Inanspruchnahme von therapeutischen Optionen (in der Kategorie "Konsistenz») beweisrechtlich als Indizien Beachtung finden [6]. Die grundsätzlich gegebene Therapierbarkeit sei demnach bei somatoformen und gleichgestellten Störungen kein Ausschlussgrund für die Bejahung einer Invalidität [7].

Weiter führte das Bundesgericht aus, dass von medizinischer Warte funktionelle Beeinträchtigungen durch somatoforme/funktionelle Störungen und durch solche depressiver Natur gleich gross seien. Für das höchste Gericht der Schweiz steht fest, dass viele depressive Erkrankungen prinzipiell durch Antidepressiva und Psychotherapie behandelbar seien, wobei offenbar bloss etwa in der Hälfte der behandelten Fälle von einer adäquaten Depressionsbehandlung nach psy- chiatrischen Standards ausgegangen werden könne. Selbst wenn in der Mehrzahl der Fälle depressive Episoden adäquat behandelt werden würden, günstig verlaufen und es zu einer vollständigen Remission oder Teilremission innert weniger Monate komme, seien dennoch trotz lege artis durchgeführter Behandlungsmassnahmen chronische Verläufe mit über zweijähriger Dauer vorstellbar, wobei komorbide Leiden die Behandlungsdauer wesentlich beeinflussen könnten [8]. Mit dem Hinweis auf eine «regelmässig gute Therapierbarkeit» bei leichten bis mittelschweren Störungen direkt auf eine fehlende invalidenversicherungsrechtlich relevante Einschränkung der Arbeitsfähigkeit zu schliessen, greife aus Sicht des Bundesgerichts daher zu kurz und blende wesentliche medizinische Aspekte dieses Krankheitsgeschehens in sachlich unbegründeter Weise aus. Die Therapierbarkeit vermag demnach keine abschliessende evidente Aussage über das Gesamtmass der Beeinträchtigung und deren Relevanz im invalidenversicherungsrechtlichen Kontext zu liefern. Einen Gesundheitsschaden allein gestützt auf das Argument der fehlenden Therapieresistenz unbesehen seiner funktionellen Auswirkungen als invalidenversicherungsrechtlich nicht relevant einzustufen, mit der Konsequenz eines Ausschlusses von Rentenleistungen, sei weder sachlich geboten noch medizinisch abgestützt [9]. Insgesamt kann festgehalten werden, dass bei sämtlichen psychischen Leiden künftig ein strukturiertes Beweisverfahren anhand der vom Bundesgericht in BGE 141 V 281 vorgegebenen Indikatoren durchgeführt 
werden muss. Diese Indikatoren beurteilen insbesondere, ob die gesundheitlichen Leiden der betroffenen Person sie derart in ihrer Funktionalität einschränken (Kategorie "Funktioneller Schweregrad») und so konsistent wirken (Kategorie «Konsistenzprüfung»), dass ein sozialversicherungsrechtlicher Anspruch auf Invalidenrente zugesprochen werden kann.

\section{Ausnahmen}

Aus Gründen der Verhältnismässigkeit kann aus Sicht des Bundesgerichts dort von einem strukturierten Beweisverfahren abgesehen werden, wo es nicht nötig oder auch gar nicht geeignet sei. Die Frage der Notwendigkeit in diesem Sinne beurteile sich nach dem konkreten Beweisbedarf. Sie fehle ganz allgemein in Fällen, die sich durch die Erhebung prägnanter Befunde und übereinstimmende fachärztliche Einschätzungen hinsichtlich Diagnose und funktioneller Auswirkungen im Rahmen beweiswertiger Arztberichte und Gutachten auszeichnen. Was die Befunde angehe, sei etwa an Störungsbilder wie Schizophrenie, Zwangs-, Ess- und Panikstörungen zu denken, die sich aufgrund klinischer psychiatrischer Untersuchung bezüglich ihrer Überprüf- und Objektivierbarkeit mit somatischen Erkrankungen vergleichen lassen können. Hier zeige sich die Beweisproblematik, wenn überhaupt, vor allem bezüglich der funktionellen Auswirkungen. Daher habe auch bei jenen Störungen eine vertiefende Prüfung hinsichtlich des funktionellen Schweregrades und insbesondere der Konsistenz zu erfolgen, wenn Hinweise auf Inkonsistenzen, auf Aggravation oder Simulation bestehen würden. Anderseits bleibe ein strukturiertes Beweisverfahren dort entbehrlich, wo im Rahmen beweiswertiger fachärztlicher Berichte eine Arbeitsunfähigkeit in nachvollziehbar begründeter Weise verneint werden und allfälligen gegenteiligen Einschätzungen mangels fachärztlicher Qualifikation oder aus anderen Gründen kein Beweiswert beigemessen werden könne [10].

\section{Welche Folgen hat die neue Rechtsprechung?}

Die aktuelle Rechtsprechung des Bundesgerichts bewirkt zuerst, dass die alleinige Diagnose einer leichtbis mittelgradigen Depression, unabhängig davon, ob sie als rezidivierend oder remittiert anzusehen ist, per se keinen Ausschlussgrund mehr bilden kann. Während noch im Jahr 2017 die Diagnose einer leicht- bis mittelgradigen Depression grundsätzlich zu keiner Gewährung eines sozialversicherungsrechtlichen Leistungsanspruchs führte, hat sich die Rechtsprechung erheblich weiterentwickelt. Entscheidend ist nun viel- mehr die Frage nach der funktionellen Auswirkung einer Störung, da die Diagnose nicht mehr im Zentrum steht, sondern Ausgangspunkt zur Beurteilung der Frage ist, ob ein Gesundheitsschaden im Sinne der klassifizierenden Merkmale überhaupt vorliegt oder nicht [11]. Zweitens steht mit der neuen Rechtsprechung auch das Verhältnis zwischen Medizin und Recht vermehrt im Fokus. Das höchste Gericht der Schweiz drückt dies so aus, dass sich sowohl die medizinischen Sachverständigen als auch die Organe der Rechtsanwendung bei ihrer Einschätzung des Leistungsvermögens an den normativen Vorgaben zu orientieren hätten; die Gutachter im Idealfall gemäss der entsprechend formulierten Fragestellung. Die Rechtsanwender hätten aus Sicht des Bundesgerichts die medizinischen Angaben frei zu überprüfen, insbesondere daraufhin, ob die Ärzte sich an die massgebenden normativen Rahmenbedingungen gehalten haben [11]. Als Konsequenz bedeutet dies, dass der Beweis für eine lang andauernde und erhebliche gesundheitsbedingte Arbeitsunfähigkeit nur dann als geleistet betrachtet werden kann, wenn die Prüfung der massgeblichen Beweisthemen im Rahmen einer umfassenden Betrachtung ein stimmiges Gesamtbild einer Einschränkung in allen Lebensbereichen (Konsistenz) für die Bejahung einer Arbeitsunfähigkeit zeigt. Fehlt es daran, ist der Beweis nicht geleistet [11].

Hinzuweisen ist ferner darauf, dass die neue Rechtslage bei den psychischen Leiden zu keiner losgelösten Prüfung anhand der Indikatoren führen darf. Oder anders ausgedrückt: Das für die Beurteilung des sozialversicherungsrechtlichen Anspruchs erstellte Gutachten darf von den Rechtsanwendern mittels der vorgegebenen Indikatoren nicht so umgedeutet werden, dass die betroffene Person an der (berechtigten) Leistungszusprache verhindert wird [12].

\section{Bildnachweis}

(c) Franky44 | Dreamstime.com

Literatur

1 BGE 140 V 193 E. 3.3; Urteil des Bundesgerichts 9C 841/2016 vom 8. Februar 2017 E. 3.1; Urteil des Bundesgerichts 9 C 539/2015 vom 21. März 2016 E. 4.1.3.1

2 Mit weiteren Hinweisen Slavik E. Invalidenrentenanspruch bei depressiven Erkrankungen. Jusletter vom 4. September 2017. N 1ff.

3 Sager R. Die bundesgerichtliche Rechtsprechung betreffend Depressionen. SZS 2015. S. 317-318.

4 BGE 143 V 418 lit. D; 143 V 409 lit. E.

5 BGE 143 V 409 E. 4.2.1; 127 V 294 E. 4b/cc.

6 Jeger J. BGE 141 V 281: Ein Sommernachtstraum oder viel Lärm um nichts? HAVE 2018. S. 151ff.; Jeger J. Die neue Rechtsprechung zu psychosomatischen Krankheitsbildern. Jusletter vom 13. Juli 2015. $\mathrm{N} 1 \mathrm{ff}$

7 BGE 143 V 409 E. 4.2.2; zum Ganzen BGE 141 V 281.

8 BGE 143 V 409 E. 4.3

9 BGE 143 V 409 E. 4.4

10 BGE 143 V 418 E. 7.1; 143 V 409 E. 4.5.3.

11 BGE 143 V 418 E. 6.

12 Zum Ganzen BGE 144 V 50. 